\title{
EVIDÊNCIAS DA EFICÁCIA DA LASERTERAPIA DE BAIXA INTENSIDADE NA PREVENÇÃO E TRATAMENTO DA MUCOSITE ORAL
}

\section{EVIDENCE OF THE EFFECTIVENESS OF LOW-LEVEL LASER THERAPY IN THE PREVENTION AND TREATMENT OF ORAL MUCOSITIS}

\author{
Gualberto de Abreu Soares ${ }^{1}$ \\ Jurecir da Silva Silva² \\ Elaine Ferreira do Nascimento ${ }^{3}$ \\ Jéssica Pereira dos Santos ${ }^{4}$ \\ Jossuely Rocha Mendes ${ }^{5}$
}

Resumo: Introdução: Dentre as formas de prevenção e controle da Mucosite Oral, o uso dos Lasers de Baixa Intensidade destaca-se como eficiente. Objetivo: Avaliar as evidências científicas do uso da LBI na prevenção e tratamento da MO. Metodologia: Realizou-se uma pesquisa eletrônica na Biblioteca Virtual em Saúde - (www.bireme.br), na qual foram selecionadas as publicações dos anos de 2003 a 2013, que possuíam como palavras-chave os termos; Mucosite, Lasers, Prevenção e controle, na língua portuguesa; e Mucositis, lasers, Prevention and control, na língua inglesa. Foram selecionados 21 artigos pelos itens de inserção: (1) pesquisas escritas no idioma inglês ou portugues; (2) pesquisas de estudos experimentais; (3) pesquisas originais. Os itens de restrição foram: 1) trabalhos científicos que estivessem divulgados em outras formatações, como revisões e materiais educativos; 2) pesquisas que não contivessem seu resumo nas bases de dados selecionadas; 3) pesquisas de acesso restrito; 4) pesquisas com deficiência na descrição metodológica. Resultados: $42 \%(n=9)$ dos artigos relatando o uso da laserterapia no tratamento da mucosite; $28 \%$ ( $n=6)$, na prevenção; e 30\% ( $n=7)$, na prevenção e tratamento. Discussão: O laser de Arseneto de Gálio e Alumínio, com comprimento de onda de 660nm, potência de $30 \mathrm{~mW}$ e densidade de energia $2 \mathrm{~J} / \mathrm{cm} 2$, aplicado de forma pontual é o mais eficiente. Conclusão: $A$ LBl é eficiente na prevenção e tratamento da Mucosite Oral. Entretanto, não existe uma padronização de protocolos de uso e, muito menos, trabalhos que busquem resolver essa particularidade.

Palavras-chave: Mucosite; lasers; prevenção e controle.

Abstract: Introduction: Among the forms of prevention and control of Oral Mucositis, the use of lowintensity Lasers stands out as efficient. Objective: to evaluate the scientific evidence of the use of LBI in the prevention and treatment of MO. Material and Method: an electronic search was performed in the Virtual Health Library - (www.bireme.br), where we have selected the publications from 2003 to 2013, which had as keywords the terms; Mucositis, Lasers, prevention and control, in the Portuguese language; and Mucositis, lasers, Prevention and control in the English language. 21 articles were selected by the insert items: (1) surveys written in the English or Portuguese languages; (2) researches of experimental studies; (3) original researches. Constraint items were: 1) scientific papers that were published in other formatting, such as reviews and educational materials; 2) researches that do not contain their abstracts in the selected

\footnotetext{
${ }^{1}$ Especialista em Saúde Pública pelo Instituto de Ensino Superior Múltiplo - IESM, Associação de Ensino Superior do Piauí - AESPI, Brasil. E-mail: gualbertoprofisio@gmail.com.

2 Especialista em Análises Clinicas pela Sociedade Brasileira de Analises Clinicas, Instituto Federal do Piauí/Fundação Oswaldo Cruz, Piauí, Brasil. E-mail: jurecir.silva@ifpi.edu.br.

3 Doutorado em Ciências pelo Instituto Fernandes Figueira Fundação Oswaldo Cruz, Fundação Oswaldo Cruz Fiocruz, Brasil. E-mail: negraelaine@gmail.com.

${ }^{4}$ Mestrado em Medicina Tropical pela Instituto Oswaldo Cruz, Fundação Oswaldo Cruz - Fiocruz, Brasil. E-mail: jessik_ssantos@hotmail.com.

5 Discente do Curso Técnico em Análises clínicas/Bacharelado em Biomedicina - Instituto Federal do Piauí/Universidade Mauricio de Nassau, Brasil. E-mail: jossuelym@hotmail.com.
} 
databases; 3) restricted access researches; 4) researches with deficiency in the methodological description. Results: $42 \%(n=9)$ of the articles reporting the use of laser therapy in the treatment of mucositis, $28 \%$ ( $n$ $=6)$ in the prevention and $30 \%(n=7)$ in the prevention and treatment. Discussion: the laser of gallium and aluminum N. gauze, with wavelength of $660 \mathrm{~nm}$, power of $30 \mathrm{~mW}$ and energy density of $2 \mathrm{~J} / \mathrm{cm} 2$, applied in a punctual way, is the most efficient. Conclusion: $L B I$ is efficient in the prevention and treatment of Oral Mucositis. However, there is no standardization of protocols nor works that aim at solving this particularity.

Keywords: Mucositis; lasers; prevention and control.

\section{INTRODUÇÃO}

A Mucosite Oral (MO) é hoje tida como a complicação não hematopoiética mais frequente no tratamento antineoplásico. Os estudos mais recentes da fisiopatologia e ocorrência da MO promoveram avanços consideráveis no seu entendimento e auxiliaram no descobrimento de técnicas de prevenção e terapias que previnam as consequências diretas da radioterapia e quimioterapia (BERGER, 2001).

Entretanto, a MO é uma patologia difícil de se controlar, não existindo, atualmente, um entendimento a respeito de um protocolo de tratamento, podendo afirmar que os existentes ainda possuem insucessos. As sequelas provocadas pela $\mathrm{MO}$ no tratamento antineoplásico são importantes, pois podem provocar, até mesmo, a sua interrupção (TROTTl et al., 2003).

Nos achados literários, não existe uma conformidade sobre a melhor forma de manejo da MO. Os manejos são diversificados e buscam atenuar os sintomas álgicos das lesões ou evitá-las. No universo de terapias propostas pela literatura, a Laserterapia de Baixa Intensidade (LBI), aplicada na mucosa oral, destaca-se como uma boa alternativa para prevenir e realizar o manejo da mucosite, pois tem bons resultados, é de baixo custo e não traumática (BENSADOUN, 2006).

A aplicação de LBI na mucosa oral é capaz de prevenir a ocorrência de $M O$ em estágios avançados de pacientes oncológicos. Em indivíduos submetidos à LBI como profilaxia, ela se mostrou cerca de nove vezes mais eficaz que sua ausência. Esses achados são diferentes de alguns dados literários que não observaram, de uma maneira geral, a eficácia da LBI na prevenção da $\mathrm{MO}$ em pacientes sob terapia antineoplásica (CRUZ et al., 2007).

A pesquisa se faz necessária para contribuir com a comunidade científica sobre a elucidação das evidências do uso LBI como prevenção e tratamento da MO. O objetivo deste trabalho é apresentar as evidências no que diz respeito aos resultados dos tratamentos, parâmetros utilizados e discrepâncias do uso da LBI na MO encontradas na literatura. 


\section{MATERIAIS E MÉTODO}

Trata-se de uma pesquisa na literatura para conseguir dados encontrados em pesquisa eletrônicas, na Biblioteca Virtual em Saúde - (www.bireme.br), em que foram selecionadas as publicações dos últimos dez anos.

A pesquisa foi desenvolvida pela busca de estudos que possuíram como palavras chaves os termos Mucosite, Lasers, Prevenção e controle, na língua portuguesa; e Mucositis, lasers, Prevention and control na língua inglesa, a fim de verificar a relação entre esses estudos.

Os estudos foram selecionados após leitura e aplicação. Itens de inserção: (1) pesquisas escritas no idioma inglês ou português; (2) pesquisas de estudos experimentais; (3) pesquisas originais. E itens de restrição: 1) trabalhos científicos que estivessem divulgados em outras formatações, configurados como revisões e materiais educativos; 2) pesquisas que não contivessem seu resumo nas bases de dados selecionadas; 3) pesquisas de acesso restrito; 4) pesquisas com deficiência na descrição metodológica, principalmente no que se referem a objetivos, métodos, resultados e conclusões. Ressalta-se a atenção observada para que os artigos não fossem incluídos duas vezes, caso estivessem indexados em mais de uma das bases de dados selecionadas.

Os estudos selecionados foram apresentados em tabelas e quadros e analisados conforme o objetivo da pesquisa.

\section{RESULTADOS}

Durante o levantamento bibliográfico, foram encontrados 31 trabalhos relevantes a serem revisados, mas, ao aplicarmos os critérios de inclusão e exclusão, restaram 21 artigos. Deles, 1 (um) artigo teve como amostra hamster; os outros 20 (vinte) artigos tiveram seres humanos como amostra, conforme a Tabela 1.

Tabela 1 - Dados gerais dos artigos revisados.

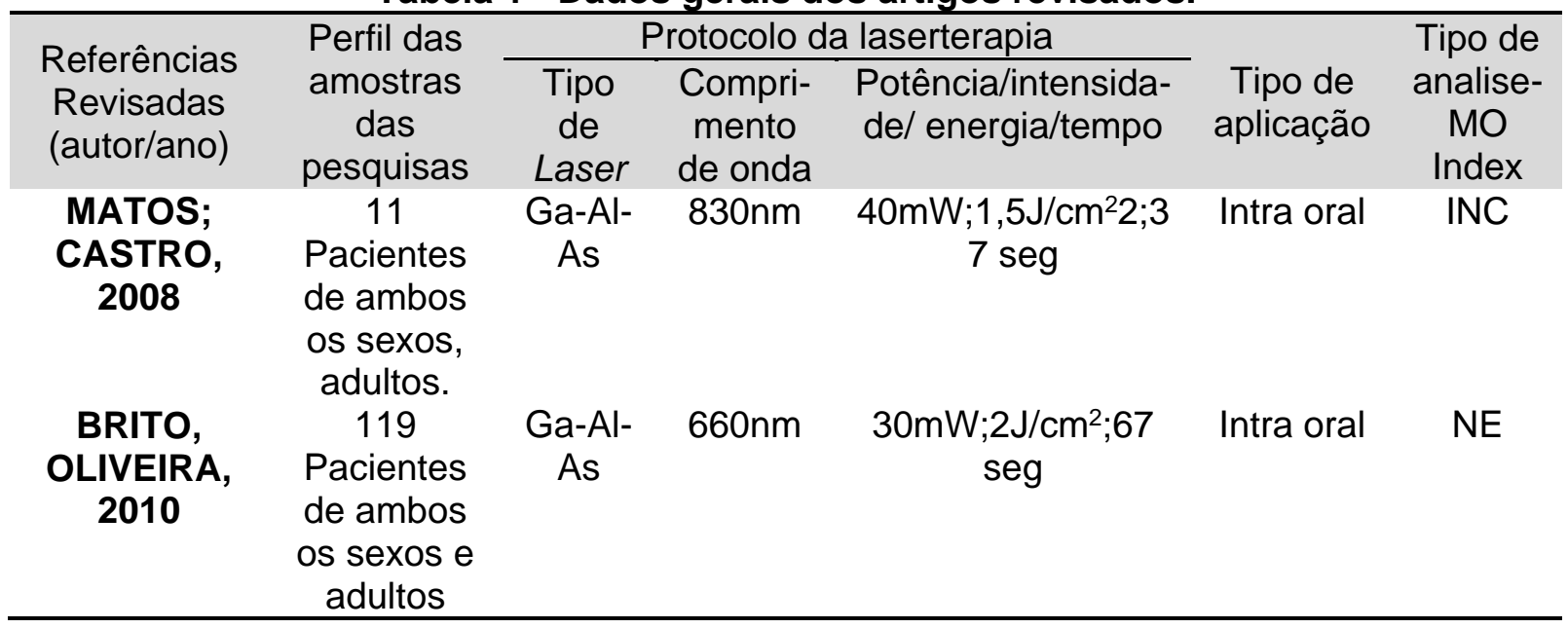




\begin{tabular}{|c|c|c|c|c|c|c|}
\hline \multirow[b]{2}{*}{$\begin{array}{l}\text { Referências } \\
\text { Revisadas } \\
\text { (autor/ano) }\end{array}$} & \multirow{2}{*}{$\begin{array}{c}\text { Perfil das } \\
\text { amostras } \\
\text { das } \\
\text { pesquisas }\end{array}$} & \multicolumn{3}{|c|}{ Protocolo da laserterapia } & \multirow[b]{2}{*}{$\begin{array}{l}\text { Tipo de } \\
\text { aplicação }\end{array}$} & \multirow{2}{*}{$\begin{array}{l}\text { Tipo de } \\
\text { analise- } \\
\text { MO } \\
\text { Index }\end{array}$} \\
\hline & & $\begin{array}{l}\text { Tipo } \\
\text { de } \\
\text { Laser }\end{array}$ & $\begin{array}{l}\text { Compri- } \\
\text { mento } \\
\text { de onda }\end{array}$ & $\begin{array}{l}\text { Potência/intensida- } \\
\text { de/ energia/tempo }\end{array}$ & & \\
\hline $\begin{array}{l}\text { AMORIM } \\
\text { et al., } 2006\end{array}$ & $\begin{array}{c}14 \\
\text { pacientes } \\
\text { de ambos } \\
\text { os sexos, } \\
\text { adultos }\end{array}$ & $\begin{array}{l}\text { Ga-Al- } \\
\text { As }\end{array}$ & $660 \mathrm{~nm}$ & 30nw: 6J/cm²; NE & Intra oral & WHO \\
\hline $\begin{array}{c}\text { CUNHA } \\
\text { et al., } 2004\end{array}$ & $\begin{array}{l}30 \\
\text { pacientes } \\
\text { de ambos } \\
\text { os sexos, } \\
\text { adultos }\end{array}$ & $\begin{array}{l}\text { Ga-Al- } \\
\text { As }\end{array}$ & $\begin{array}{c}685 \mathrm{~nm} \\
\mathrm{e} \\
830 \mathrm{~nm}\end{array}$ & $\begin{array}{c}35 \mathrm{~mW} \text { e } \\
70 \mathrm{~mW} / 4 \mathrm{~J} / \mathrm{cm} 2 ; \mathrm{NE}\end{array}$ & Intra oral & RTOG \\
\hline $\begin{array}{l}\text { BRITO } \\
\text { et al., } 2012\end{array}$ & $\begin{array}{c}74 \\
\text { pacientes } \\
\text { de ambos } \\
\text { os sexos, } \\
\text { adultos }\end{array}$ & $\begin{array}{l}\text { Ga-Al- } \\
\text { As }\end{array}$ & $660 \mathrm{~nm}$ & $\begin{array}{c}30 \mathrm{~mW} / 2 \mathrm{~J} / \mathrm{cm} 2 ; 69 \\
\text { seg }\end{array}$ & Intra oral & OMS \\
\hline $\begin{array}{l}\text { LINO } \\
\text { et al., } 2011\end{array}$ & $\begin{array}{l}1 \text { paciente } \\
\text { adulto. }\end{array}$ & $\begin{array}{l}\text { Ga-Al- } \\
\text { As }\end{array}$ & $660 \mathrm{~nm}$ & $\begin{array}{c}40 \mathrm{~mW} ; 2,4 \mathrm{~J} / \mathrm{cm}^{2} \\
\text { por pontual e } 14,4 \\
\mathrm{~J} / \mathrm{cm}^{2} \text { por sessão; } \\
\mathrm{NE}\end{array}$ & Intra oral & WHO \\
\hline $\begin{array}{l}\text { LIMA } \\
\text { et al., } 2010\end{array}$ & $\begin{array}{c}25 \\
\text { pacientes } \\
\text { adultos de } \\
\text { ambos os } \\
\text { sexos. }\end{array}$ & $\begin{array}{l}\text { Ga-Al- } \\
\text { As }\end{array}$ & $830 \mathrm{~nm}$ & $\begin{array}{c}60 \mathrm{~mW} ; 2,4 \mathrm{~J} / \mathrm{cm}^{2} ; \\
\mathrm{NE}\end{array}$ & Intra oral & OTS \\
\hline $\begin{array}{l}\text { DJAVID } \\
\text { et al., } 2001\end{array}$ & $\begin{array}{c}55 \\
\text { pacientes } \\
\text { adultos de } \\
\text { ambos os }\end{array}$ & $\begin{array}{l}\text { Ga-Al- } \\
\text { As }\end{array}$ & $630 \mathrm{~nm}$ & $30 \mathrm{~mW} ; 5 \mathrm{~J} / \mathrm{cm}^{2} ; \mathrm{NE}$ & Intra oral & WHO \\
\hline $\begin{array}{l}\text { LOPES } \\
\text { et al., } 2006\end{array}$ & $\begin{array}{c}\text { sexos. } \\
60 \\
\text { pacientes } \\
\text { de ambos }\end{array}$ & $\underset{P}{\ln G a A l}$ & $685 \mathrm{~nm}$ & $\begin{array}{c}35 \mathrm{~mW} ; 2,0 \mathrm{~J} / \mathrm{cm}^{2} \\
58 \mathrm{seg} .\end{array}$ & Intra oral & INC \\
\hline & $\begin{array}{l}\text { os sexos e } \\
\text { adultos }\end{array}$ & & & & & \\
\hline $\begin{array}{l}\text { KELNER; } \\
\text { CASTRO, } \\
2007\end{array}$ & $\begin{array}{c}2 \\
\text { pacientes, } \\
\text { ambos os } \\
\text { sexos, } \\
\text { adulto }\end{array}$ & $\underset{P}{\ln G a A l}$ & $685 \mathrm{~nm}$ & $\begin{array}{c}35 \mathrm{~mW} ; 1,1 \mathrm{~J} / \mathrm{cm}^{2} 2 ; 4 \\
0 \mathrm{seg}\end{array}$ & Intra oral & NE \\
\hline $\begin{array}{c}\text { LOPEZ } \\
\text { et al., } 2013\end{array}$ & 96 hamster & $\underset{P}{\ln G a A l}$ & $660 \mathrm{~nm}$ & $\begin{array}{c}40 \mathrm{~mW} ; 6,6 \mathrm{~J} / \\
\mathrm{cm}^{2} ; \mathrm{NE}\end{array}$ & Intra oral & $\begin{array}{l}\text { Lima et } \\
\text { al. }\end{array}$ \\
\hline $\begin{array}{l}\text { ANTUNES } \\
\text { et al., } 2007\end{array}$ & $\begin{array}{c}38 \\
\text { pacientes } \\
\text { adultos de } \\
\text { ambos os } \\
\text { sexos }\end{array}$ & $\underset{P}{\ln G a A l}$ & $660 \mathrm{~nm}$ & $\begin{array}{c}50 \mathrm{mw} ; 4 \mathrm{~J} / \mathrm{cm}^{2} \\
16,7 \mathrm{seg}\end{array}$ & Intra oral & $\begin{array}{l}\text { WHO e } \\
\text { OMAS }\end{array}$ \\
\hline
\end{tabular}




\begin{tabular}{|c|c|c|c|c|c|c|}
\hline \multirow[b]{2}{*}{$\begin{array}{l}\text { Referências } \\
\text { Revisadas } \\
\text { (autor/ano) }\end{array}$} & \multirow{2}{*}{$\begin{array}{l}\text { Perfil das } \\
\text { amostras } \\
\text { das } \\
\text { pesquisas }\end{array}$} & \multicolumn{3}{|c|}{ Protocolo da laserterapia } & \multirow[b]{2}{*}{$\begin{array}{l}\text { Tipo de } \\
\text { aplicação }\end{array}$} & \multirow{2}{*}{$\begin{array}{l}\text { Tipo de } \\
\text { analise- } \\
\text { MO } \\
\text { Index }\end{array}$} \\
\hline & & $\begin{array}{l}\text { Tipo } \\
\text { de } \\
\text { Laser }\end{array}$ & $\begin{array}{l}\text { Compri- } \\
\text { mento } \\
\text { de onda }\end{array}$ & $\begin{array}{l}\text { Potência/intensida- } \\
\text { de/ energia/tempo }\end{array}$ & & \\
\hline $\begin{array}{l}\text { CARVALHO } \\
\text { et al., } 2011\end{array}$ & $\begin{array}{l}70 \\
\text { pacientes } \\
\text { adultos de } \\
\text { ambos os } \\
\text { sexos. }\end{array}$ & $\begin{array}{l}\text { InGaAl } \\
P\end{array}$ & $660 \mathrm{~nm}$ & $\begin{array}{c}15 \mathrm{~mW} \text { e } 5 \mathrm{~mW} \\
3,8 \mathrm{~J} / \mathrm{cm}^{2} \mathrm{e} \\
1,3 \mathrm{~J} / \mathrm{cm}^{2} ; 10 \mathrm{seg} .\end{array}$ & Intra oral & $\begin{array}{l}\text { INC e } \\
\text { WHO }\end{array}$ \\
\hline $\begin{array}{l}\text { ANTUNES, } \\
\text { et al., } 2008\end{array}$ & $\begin{array}{l}11 \\
\text { pacientes } \\
\text { adultos de } \\
\text { ambos os } \\
\text { sexos }\end{array}$ & $\underset{P}{\operatorname{InGaAl}}$ & $660 \mathrm{~nm}$ & $\begin{array}{c}50 \mathrm{~mW} ; 8 \mathrm{~J} / \mathrm{cm}^{2} \\
33,4 \mathrm{seg} .\end{array}$ & Intra oral. & $\begin{array}{l}\text { WHO e } \\
\text { OMAS }\end{array}$ \\
\hline $\begin{array}{l}\text { KHOURI } \\
\text { et al., } 2009\end{array}$ & $\begin{array}{l}22 \\
\text { pacientes } \\
\text { adultos de } \\
\text { ambos os } \\
\text { sexos }\end{array}$ & $\begin{array}{l}\text { InGaAl } \\
\mathrm{Pe} \\
\text { Ga-Al- } \\
\text { As }\end{array}$ & $\begin{array}{l}660 \mathrm{~nm} \\
\mathrm{e} \\
780 \mathrm{~nm}\end{array}$ & $\begin{array}{c}25 \mathrm{~mW} ; 6,3 \mathrm{~J} / \mathrm{m}^{2} \\
10 \mathrm{seg} .\end{array}$ & Intra oral & $\begin{array}{l}\text { WHO e } \\
\text { OMAS }\end{array}$ \\
\hline $\begin{array}{c}\text { FANI } \\
\text { et al., } 2013\end{array}$ & $\begin{array}{l}40 \\
\text { pacientes } \\
\text { com } 12 \\
\text { anos ou } \\
\text { mais. }\end{array}$ & AIGaln & $660 \mathrm{~nm}$ & $\begin{array}{c}25 \mathrm{~mW} ; 1,5 \mathrm{~J} / \mathrm{cm}^{2} \\
1 \mathrm{~min} .\end{array}$ & Intra oral & WHO \\
\hline $\begin{array}{c}\text { MAIYA } \\
\text { et al., } 2006\end{array}$ & $\begin{array}{c}50 \\
\text { pacientes } \\
\text { de ambos }\end{array}$ & $\mathrm{He}-\mathrm{Ne}$ & $620 \mathrm{~nm}$ & NE & Intra oral & NE \\
\hline $\begin{array}{l}\text { SANDOVAL } \\
\text { et al., } 2003\end{array}$ & $\begin{array}{l}\text { os sexos } \\
18 \\
\text { pacientes } \\
\text { adultos de } \\
\text { ambos os } \\
\text { sexos. }\end{array}$ & NE & $660 \mathrm{~nm}$ & $\begin{array}{c}30 \mathrm{~mW} ; 2 \mathrm{~J} / \mathrm{cm}^{2} ; 66 \\
\text { seg. }\end{array}$ & Intra oral & INC \\
\hline $\begin{array}{l}\text { ARBABI- } \\
\text { KALATI } \\
\text { et al., } 2013\end{array}$ & $\begin{array}{l}48 \\
\text { pacientes } \\
\text { adultos de } \\
\text { ambos os } \\
\text { sexos }\end{array}$ & NE & $630 \mathrm{~nm}$ & $30 \mathrm{~mW}: 5 \mathrm{~J} / \mathrm{cm}^{2} ; \mathrm{NE}$ & Intra oral & WHO \\
\hline $\begin{array}{l}\text { MEDEIROS } \\
\text { et al. } 2013\end{array}$ & $\begin{array}{l}1 \text { paciente } \\
\text { pediátrico } \\
\text { fe } \\
\text { minino. }\end{array}$ & NE & $\begin{array}{l}780 \mathrm{~nm} \\
\mathrm{e} \\
660 \mathrm{~nm}\end{array}$ & $\begin{array}{c}4,3 \mathrm{~J} / \mathrm{cm}^{2} \text { (com } \\
\text { efeito analgésico e } \\
\text { cicatrizante) e } \\
1,3 \mathrm{~J} / \mathrm{cm}^{2} \text { (com } \\
\text { efeito preventivo); }\end{array}$ & Intra oral & OMS \\
\hline $\begin{array}{c}\text { SILVA } \\
\text { et al., } 2010\end{array}$ & $\begin{array}{c}16 \\
\text { pacientes } \\
\text { escolhidos } \\
\text { de forma } \\
\text { aleatória. }\end{array}$ & NE & $780 \mathrm{~nm}$ & $\begin{array}{c}5,0 \mathrm{~J} / \mathrm{cm}^{2} \text { (com } \\
\text { efeito preventivo }\end{array}$ & Intra oral & OMS \\
\hline
\end{tabular}

Notas: NE : não especificado; VAS: visual analogue scale; WHO: World Health Organization; OMAS: Oral Mucositis Assessment Scale; OMI-A and OMI--B Oral/ ECOG: Oral Mucositis Index Scale and the Eastern Cooperative Oncology Group; INC: Instituto Nacional de Câncer; (7) RTGO: Radiation Therapy Oncology Group. 
Vários estudos clínicos com diferentes protocolos foram realizados com 0 objetivo de comprovar a eficácia do LBI no manejo (tratamento, prevenção ou ambos) da mucosite bucal ${ }^{15}$. Verifica-se, nos trabalhos da literatura revisada, uma multiplicidade de protocolos e tipos de lasers utilizados no tratamento da MO (tabela 2 e gráfico 1).

Tabela 2 - Distribuição dos protocolos aplicados com laserterapia de baixa intensidade dos artigos pesquisados com relação ao tipo de manejo da mucosite oral.

\begin{tabular}{|c|c|c|c|c|}
\hline \multirow{2}{*}{$\begin{array}{l}\text { Tipo de } \\
\text { manejo }\end{array}$} & \multirow{2}{*}{$\begin{array}{l}\text { Referência Revisada } \\
\text { (autor/ano) }\end{array}$} & \multicolumn{3}{|c|}{$\begin{array}{l}\text { Protocolo de laserterapia de baixa } \\
\text { intensidade }\end{array}$} \\
\hline & & $\begin{array}{c}\text { Comprimento } \\
\text { de onda }\end{array}$ & $\begin{array}{l}\text { Densidade } \\
\text { de energia }\end{array}$ & Potência \\
\hline \multirow{9}{*}{ Tratamento } & $\begin{array}{l}\text { CUNHA } \\
\text { et al. } 2004\end{array}$ & 685 e $830 \mathrm{~nm}$ & $4 \mathrm{~J} / \mathrm{cm}^{2}$ & $\begin{array}{c}35 \mathrm{e} \\
70 \mathrm{~mW}\end{array}$ \\
\hline & $\begin{array}{l}\text { BRITO } \\
\text { et al.,2012 }\end{array}$ & $660 \mathrm{~nm}$ & $2,0 \mathrm{~J} / \mathrm{cm}^{2}$ & $30 \mathrm{~mW}$ \\
\hline & $\begin{array}{l}\text { MATOS; CASTRO. } \\
2008\end{array}$ & $830 \mathrm{~nm}$ & $1,5 \mathrm{~J} / \mathrm{cm}^{2}$ & $40 \mathrm{~mW}$ \\
\hline & $\begin{array}{l}\text { SANDOVAL, } \\
\text { et al., } 2003\end{array}$ & $660 \mathrm{~nm}$ & $2,0 \mathrm{~J} / \mathrm{cm}^{2}$ & $30 \mathrm{~mW}$ \\
\hline & $\begin{array}{l}\text { ANTUNES, } \\
\text { et al., } 2008\end{array}$ & $660 \mathrm{~nm}$ & $8 \mathrm{~J} / \mathrm{cm}^{2}$ & $50 \mathrm{~mW}$ \\
\hline & $\begin{array}{l}\text { LINO } \\
\text { et al., } 2011\end{array}$ & $660 \mathrm{~nm}$ & $2,4 \mathrm{~J} / \mathrm{cm}^{2}$ & $40 \mathrm{~mW}$ \\
\hline & $\begin{array}{l}\text { LOPES } \\
\text { et al., } 2006\end{array}$ & $685 \mathrm{~nm}$ & $2,0 \mathrm{~J} / \mathrm{cm}^{2}$ & $35 \mathrm{~mW}$ \\
\hline & $\begin{array}{l}\text { MEDEIROS } \\
\text { et al., } 2013\end{array}$ & 780 a $660 \mathrm{~nm}$ & $\begin{array}{c}1,3 \mathrm{a} \\
4,3 \mathrm{~J} / \mathrm{cm}^{2}\end{array}$ & NE \\
\hline & $\begin{array}{l}\text { BRITO } \\
\text { et al., } 2012\end{array}$ & $660 \mathrm{~nm}$ & $2,0 \mathrm{~J} / \mathrm{cm}^{2}$ & $30 \mathrm{~mW}$ \\
\hline \multirow{5}{*}{ Prevenção } & $\begin{array}{l}\text { DJAVID } \\
\text { et al., } 2011\end{array}$ & $630 \mathrm{~nm}$ & $5 \mathrm{~J} / \mathrm{cm}^{2}$ & $30 \mathrm{~mW}$ \\
\hline & $\begin{array}{l}\text { ANTUNES } \\
\text { et al., } 2007\end{array}$ & $660 \mathrm{~nm}$ & $4 \mathrm{~J} / \mathrm{cm}^{2}$ & $50 \mathrm{~mW}$ \\
\hline & $\begin{array}{l}\text { ARBABI-KALATI } \\
\text { et al., } 2013\end{array}$ & $630 \mathrm{~nm}$ & $5 \mathrm{j} / \mathrm{cm}^{2}$ & $30 \mathrm{~mW}$ \\
\hline & $\begin{array}{l}\text { LIMA } \\
\text { et al., } 2010\end{array}$ & $830 \mathrm{~nm}$ & $2,4 \mathrm{~J} / \mathrm{cm}^{2}$ & $60 \mathrm{~mW}$ \\
\hline & $\begin{array}{l}\text { SANTOS } \\
\text { et al., } 2010\end{array}$ & $780 \mathrm{~nm}$ & $5,0 \mathrm{~J} / \mathrm{cm}^{2}$ & NE \\
\hline \multirow{6}{*}{$\begin{array}{c}\text { Tratamento } \\
\text { e } \\
\text { Prevenção }\end{array}$} & $\begin{array}{l}\text { AMORIM } \\
\text { et al., } 2006\end{array}$ & $660 \mathrm{~nm}$ & $6 \mathrm{~J} / \mathrm{cm}^{2}$ & $30 \mathrm{~mW}$ \\
\hline & $\begin{array}{c}\text { KELNER; CASTRO, } \\
2007\end{array}$ & $685 \mathrm{~nm}$ & $1 \mathrm{~J} / \mathrm{cm}^{2}$ & $35 \mathrm{~mW}$ \\
\hline & $\begin{array}{l}\text { CARVALHO } \\
\text { et al., } 2011\end{array}$ & $660 \mathrm{~nm}$ & $3,8 \mathrm{a}, 2 \mathrm{~J} / \mathrm{cm}^{2}$ & $15 \mathrm{~mW}$ \\
\hline & $\begin{array}{l}\text { KHOURI } \\
\text { et al., } 2009\end{array}$ & $660 \mathrm{~nm}$ & $6,3 \mathrm{~J} / \mathrm{cm}^{2}$ & $780 \mathrm{~mW}$ \\
\hline & $\begin{array}{l}\text { MAIYA } \\
\text { et al., } 2006\end{array}$ & $620 \mathrm{~nm}$ & $1,8 \mathrm{~J} / \mathrm{cm}^{2}$ & $10 \mathrm{~mW}$ \\
\hline & $\begin{array}{l}\text { LOPEZ } \\
\text { et al., } 2013\end{array}$ & $660 \mathrm{~nm}$ & $6,6 \mathrm{~J} / \mathrm{cm}^{2}$ & $40 \mathrm{~mW}$ \\
\hline
\end{tabular}




\begin{tabular}{l|c|c|c|c}
\hline \multirow{2}{*}{$\begin{array}{c}\text { Tipo de } \\
\text { manejo }\end{array}$} & $\begin{array}{c}\text { Referência Revisada } \\
\text { (autor/ano) }\end{array}$ & \multicolumn{3}{|c}{$\begin{array}{c}\text { Protocolo de laserterapia de baixa } \\
\text { intensidade }\end{array}$} \\
\cline { 3 - 5 } & $\begin{array}{c}\text { Comprimento } \\
\text { de onda }\end{array}$ & $\begin{array}{c}\text { Densidade } \\
\text { de energia }\end{array}$ & Potência \\
\cline { 2 - 5 } & et al., 2013 & $660 \mathrm{~nm}$ & $1,5 \mathrm{~J} / \mathrm{cm}^{2}$ & $25 \mathrm{~mW}$ \\
\hline
\end{tabular}

Fonte: Pesquisa direta

Gráfico 1 - Porcentagem dos lasers utilizados nos trabalhos revisados.

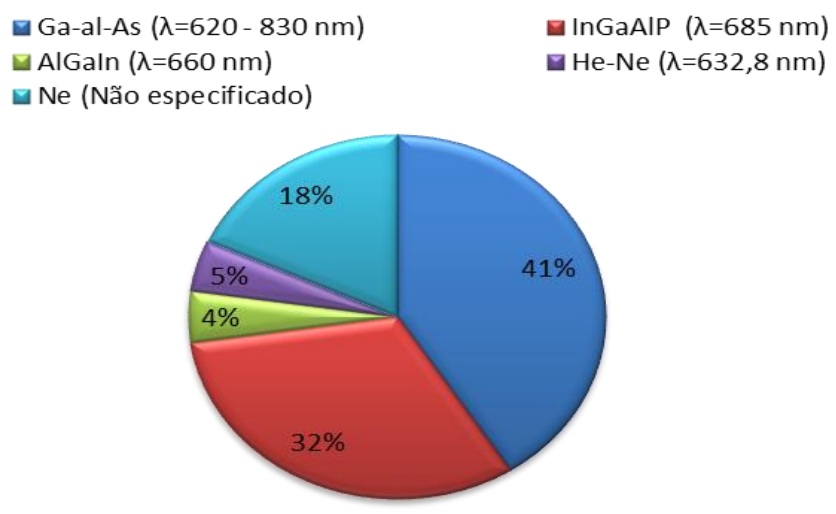

Fonte: Pesquisa direta

Levando em consideração o objetivo da pesquisa, foram encontrados, em $42 \%$ $(n=9)$ dos artigos, o relato do uso da laserterapia no tratamento da mucosite; $28 \%(n=6)$, na prevenção; e $30 \%(n=7)$, na prevenção e tratamento (Tabela 2).

Na literatura revisada, foi observado que 100\% $(n=21)$ dos artigos trazem, em suas conclusões, citações positivas em relação ao objetivo proposto, seja ele apenas o tratamento da mucosite, apenas a prevenção ou ambos.

Os resultados da pesquisa não foram satisfatórios ao considerarmos o protocolo da aplicação da laserterapia e o objetivo das pesquisas revisadas, pois não conseguimos observar um padrão nas dosimetrias utilizadas (Tabela 3).

Tabela 3 - Relação do objetivo das pesquisas e a dosimetria utilizada.

\begin{tabular}{c|ccc}
\hline \multirow{2}{*}{$\begin{array}{c}\text { Objetivo geral das } \\
\text { pesquisas revisadas }\end{array}$} & \multicolumn{3}{|c}{ Dosimetria da laserterapia utilizada nas pesquisas revisadas } \\
\cline { 2 - 4 } & $\begin{array}{c}\text { Comprimento de } \\
\text { onda }\end{array}$ & $\begin{array}{c}\text { Densidade de } \\
\text { energia }\end{array}$ & Potência \\
\hline \multirow{5}{*}{ Tratamento } & 685 e $830 \mathrm{~nm}$ & $4 \mathrm{~J} / \mathrm{cm}^{2}$ & $35 \mathrm{~mW} \mathrm{e}$ \\
& $660 \mathrm{~nm}$ & $2,0 \mathrm{~J} / \mathrm{cm}^{2}$ & $70 \mathrm{~mW}$ \\
& $830 \mathrm{~nm}$ & $1,5 \mathrm{~J} / \mathrm{cm}^{2}$ & $30 \mathrm{~mW}$ \\
& $660 \mathrm{~nm}$ & $2,0 \mathrm{~J} / \mathrm{cm}^{2}$ & $40 \mathrm{~mW}$ \\
& $660 \mathrm{~nm}$ & $8 \mathrm{~J} / \mathrm{cm}^{2}$ & $30 \mathrm{~mW}$ \\
& $660 \mathrm{~nm}$ & $2,4 \mathrm{~J} / \mathrm{cm}^{2}$ & $40 \mathrm{~mW}$ \\
& $685 \mathrm{~nm}$ & $2,0 \mathrm{~J} / \mathrm{cm}^{2}$ & $35 \mathrm{~mW}$ \\
& 780 a $660 \mathrm{~nm}$ & $1,3 \mathrm{a} 4,3 \mathrm{~J} / \mathrm{cm}^{2}$ & $\mathrm{NE}$ \\
& $660 \mathrm{~nm}$ & $2,0 \mathrm{~J} / \mathrm{cm}^{2}$ & $30 \mathrm{~mW}$ \\
\hline
\end{tabular}




\begin{tabular}{c|ccc}
\hline \multirow{2}{*}{$\begin{array}{c}\text { Objetivo geral das } \\
\text { pesquisas revisadas }\end{array}$} & \multicolumn{3}{|c}{ Dosimetria da laserterapia utilizada nas pesquisas revisadas } \\
\cline { 2 - 4 } & $\begin{array}{c}\text { Comprimento de } \\
\text { onda }\end{array}$ & $\begin{array}{c}\text { Densidade de } \\
\text { energia }\end{array}$ & Potência \\
\hline \multirow{4}{*}{ Prevenção } & $630 \mathrm{~nm}$ & $5 \mathrm{~J} / \mathrm{cm}^{2}$ & $30 \mathrm{~mW}$ \\
& $660 \mathrm{~nm}$ & $4 \mathrm{~J} / \mathrm{cm}^{2}$ & $50 \mathrm{~mW}$ \\
& $630 \mathrm{~nm}$ & $5 \mathrm{j} / \mathrm{cm}^{2}$ & $30 \mathrm{~mW}$ \\
& $830 \mathrm{~nm}$ & $2,4 \mathrm{~J} / \mathrm{cm}^{2}$ & $60 \mathrm{~mW}$ \\
& $780 \mathrm{~nm}$ & $5,0 \mathrm{~J} / \mathrm{cm}^{2}$ & $\mathrm{NE}$ \\
\hline \multirow{3}{*}{ Tratamento e } & $660 \mathrm{~nm}$ & $6 \mathrm{~J} / \mathrm{cm}^{2}$ & $30 \mathrm{~mW}$ \\
Prevenção & $685 \mathrm{~nm}$ & $1 \mathrm{~J} / \mathrm{cm}^{2}$ & $35 \mathrm{~mW}$ \\
& $660 \mathrm{~nm}$ & $3,8 \mathrm{a}, 2 \mathrm{~J} / \mathrm{cm}^{2}$ & $15 \mathrm{~mW}$ \\
& $660 \mathrm{~nm}$ & $6,3 \mathrm{~J} / \mathrm{cm}^{2}$ & $780 \mathrm{~mW}$ \\
& $620 \mathrm{~nm}$ & $1,8 \mathrm{~J} / \mathrm{cm}^{2}$ & $10 \mathrm{~mW}$ \\
& $660 \mathrm{~nm}$ & $6,6 \mathrm{~J} / \mathrm{cm}^{2}$ & $40 \mathrm{~mW}$ \\
& $660 \mathrm{~nm}$ & $1,0 \mathrm{~J} / \mathrm{cm}^{2}$ & $20 \mathrm{~mW}$ \\
\hline
\end{tabular}

Fonte: Pesquisa direta

\section{DISCUSSÃO}

Os estudos aqui revisados são complexos, pois é difícil comparar trabalhos tão diferentes entre si quanto a protocolos e parâmetros utilizados, conforme apresentação nos resultados. Chow e Barnsley (2005), numa revisão sistemática a respeito do uso da LBI para tratamento de dores cervicais, relataram a mesma marcante heterogeneidade entre os estudos, principalmente em relação aos parâmetros de utilização do laser, dose, técnica de aplicação e frequência de tratamento.

Quanto ao aparelho de laser usado nas pesquisas revisadas, encontramos o GaAIAs como o mais utilizado, porém não se verificaram justificativas para o ocorrido. Segundo Walsh (1997), esse aparelho adquiriu muita popularidade desde os anos 90 por ser pequeno, leve, de custo razoável, com potências máximas de $20 \mathrm{~mW}$, ter pontas intra orais esterilizáveis e por possuir uma luz guia vermelha. Talvez essa seja uma justificativa para o uso GaAlAs em $41 \%$ das pesquisas.

Ao observarmos as conclusões dos artigos selecionados, constatamos quase que uma unanimidade em afirmar que a LBI tem eficácia na prevenção e tratamento da MO, apenas Matos e Castro (2008) relatam que o número de pacientes tratados foi pequeno e, por isso, sugerem análises posteriores com uma amostragem maior para confirmar a eficácia do tratamento.

Araújo, Padilha e Baldisserotto (2009), realizaram um estudo com o intuito de verificar a melhoria de vida de pacientes com câncer de cabeça e pescoço e relacionála à saúde bucal. Eles concluíram que apenas a avaliação da saúde bucal é insuficiente para relacionar a qualidade de vida. Entretanto, Lima et al (2010), ao analisar 25 pacientes com câncer de cabeça e pescoço que foram tratados com LBI para prevenção da MO relatam uma melhoria no padrão de vida. É importante ressaltar que apenas este 
de todos os trabalhos desta revisão avaliaram de maneira metodológica esse item (qualidade de vida).

Segundo a literatura, não podemos padronizar um protocolo de dosimetria da LBI no manejo da $\mathrm{MO}$, mas se confrontarmos os tipos de pesquisas e o número de amostras, o uso do Ga-Al-As, com um comprimento de onda de $660 \mathrm{~nm}$, potência de $30 \mathrm{~mW}$, densidade de energia $2 \mathrm{~J} / \mathrm{cm}^{2}$, aplicado de forma pontual, é o mais eficiente (LINO et al., 2011; BRITO e OLIVEIRA., 2010; SANTOS et al., 2006).

$\mathrm{Na}$ busca de uniformizar parâmetros e mostrar efeitos positivos do laser, Lowe et al (1998), em seu estudo, sugeriu que mais estudos histológicos deveriam ser realizados, pelos quais a histologia de rotina determinasse tipos celulares, proliferação celular e vascular, produção de colágeno e respostas inflamatórias de tecidos tratados por LBI. Mas em nenhum dos trabalhos revisados observou-se a sugestão Lowe e colaboradores. Assim, na literatura presente, a clínica foi soberana em analisar o tratamento da MO com a LBI.

Infelizmente, um dos problemas do uso LBI são as discrepâncias entre as dosimetrias utilizadas nos tratamentos e pesquisas. Podemos observar isso quando comparamos Lima et al (2010), que realizaram um estudo prospectivo, comparativo e não-randomizado, com 25 pacientes, em que o objetivo era o uso da LBI como forma preventiva da $\mathrm{MO}$, a dosimetria teve como comprimento de onda $830 \mathrm{~nm}$, densidade $2,4 \mathrm{~J} / \mathrm{cm}^{2}$ e potência de $60 \mathrm{~mW}$ e Djavid et al (2011) fizeram um estudo de ensaio clínico duplo-cego randomizado placebo, em que foram analisados 16 pacientes, neles foram usados a LBI com comprimento de $630 \mathrm{~nm}$, densidade de $5 \mathrm{~J} / \mathrm{cm}^{2}$ e potência de $30 \mathrm{~mW}$ e os dois pesquisadores, apesar das diferenças de dosimetria, obtiveram os mesmos resultados.

$\mathrm{Na}$ literatura estudada e em geral, podemos encontrar uma vasta proposta de condutas a prevenção e tratamento da mucosite e com uma forte tendência a eleger a LBI como uma das mais eficácias. Ribeiro, Borda e Guimarães (2010) realizaram uma revisão de literatura das várias formas de condutas à prevenção e tratamento da mucosite e concluíram que, na falta de um modelo ideal, deve-se fazer um atendimento multidisciplinar. Assim, na multidisciplinaridade de condutas e segundo a literatura, deveríamos incorporar a LBI como uma das intervenções na prevenção e tratamento da MO.

\section{CONCLUSÃO}

Os achados desta revisão literária nos leva a afirmar que a LBI tem evidência na resolubilidade da $\mathrm{MO}$ oriunda de tratamentos radioterápicos e quimioterápicos e a resolubilidade pode ser na prevenção, no tratamento ou em ambos.

Um importante ponto a considerar na adoção da LBI no tratamento da MO é que de acordo com os artigos revisados, não existem evidências na padronização de 
dosimetria da LBI. Mas observamos uma tendência no uso do laser de Arsenieto de gálio e alumínio (Ga-Al-As), com um comprimento de onda de $660 \mathrm{~nm}$, potência de $30 \mathrm{~mW}$, densidade de energia $2 \mathrm{~J} / \mathrm{cm}^{2}$ para a resolubilidade da MO.

\section{REFERÊNCIAS}

AMORIM, José Claudio Faria et al. Laser de Baixa Intensidade no Tratamento de Mucosites em Pacientes sob Quimioterapia. Rev. Bras. Oncologia Clínica, v. 3, n. 7, p. 33-36, 2006.

ANTUNES, Héliton Spíndola et al. Low-power laser in the prevention of induced oral mucositis in bone marrow transplantation patients: a randomized trial. Blood, v. 109, n. 5, p. 2250-2255, 2007.

ANTUNES, Heliton Spindola et al. The Impact of low power laser in the treatment of conditioning-induced oral mucositis: a report of 11 clinical cases and their review.

Medicina Oral, Patologia Oral y Cirugia Bucal, v. 13, n. 3, p. E189-192, 2008.

ARAÚJO, Silvânia Suely Caribé de; PADILHA, Dalva Maria Pereira;

BALDISSEROTTO, Julio. Avaliação da condição de saúde bucal e da qualidade de vida de pacientes com câncer de cabeça e pescoço atendidos em um hospital público de Porto Alegre. Rev Bras Cancerol, v. 55, n. 2, p. 129-138, 2009.

ARBABI-KALATI, Farshid; ARBABI-KALATI, Fatemeh; MORIDI, Tahora. Evaluation of the effect of low level laser on prevention of chemotherapy-induced 20. mucositis. Acta Medica Iranica, v. 51, n. 3, p. 157-162, 2013.

BENSADOUN, R.J. Comentary. Low level LASER therapy (LLLT): A new paradigm in the management of cancer therapy-induced mucositis? Indian J Med Res. 2006, v. 124 , p. $375-378$.

BENSADOUN, J.R. Low level laser therapy (LLLT): A new paradigm in the management of cancer therapy-induced mucositis? Indian J Med Res. 2006 Oct; 124(4):375-8. Comment on: Indian J Med Res. 2006; v. 124, n. 4, p. 399-402.

BERGER, A.M., KILROY, T.J. Oral complications. In: DeVita VT, Hellman S, Roseberg SA, editors. Cancer: Principles and practice of oncology. 6th ed. Philadelphia: Lippincott Williams \& Wilkins; 2001. p. 2881-2893.

BRITO, Caroline Argolo et al. Efeito da clorexidina e do laser de baixa potência na prevenção e no tratamento da mucosite oral. Rev Odontol UNESP, v. 41, n. 4, p. 236$241,2012$.

BRITO, Caroline Argolo; OLIVEIRA, Márcio Campos. Efeito do Laser de Baixa Potência no Tratamento da Mucosite Oral. Anais da Jornada de Odontologia da Universidade Estadual de Feira de Santana, 2010. Disponível em:

Chromeextension://oemmndcbldboiebfnladdacbdfmadadm/http://www2.uefs.br/jouefs/2 010/files/anaisjouefs2010.pdf. Acesso em: 25 jul. 2015. 
CARVALHO, P. A. G. et al. Evaluation of low-level laser therapy in the prevention and treatment of radiation-induced mucositis: a double-blind randomized study in head and neck cancer patients. Oral oncology, v. 47, n. 12, p. 1176-1181, 2011.

CHOW, R.T.; BARNSLEY, L. Systematic review of the literature of low-level laser therapy (LLLT) in the management of neck pain. Lasers Surg Med, v. 37, p. 46-52, 2005.

CRUZ, L.B.; RIBEIRO, A.S., RECH, A.; ROSA, L.G.; CASTRO, C.G. \& BRUNETTO, A.L. (2007). Influence of low-energy laser in the prevention of oral mucositis in children with cancer receiving chemotherapy. Pediatric Blood and Cancer, v. 48, n. 4, p. 435440.

CUNHA, Milene Alves et al. Laser terapêutico: opção viável no tratamento da mucosite oral e xerostomia em pacientes submetidos à radioterapia e quimioterapia. In: IV Encontro Latino Americano de Pós-Gradução. 4, 2004, São José dos Campos, Anais...São José dos Campos: UNIVAP, 2004. Disponível em: <http://www.inicepg.univap.br/cd/INIC_2004/trabalhos/epg/pdf/EPG4-18.pdf>. Acesso em: 4 maio 2018.

DJAVID, Gholamreza Esmaeeli et al. Low level laser therapy in management of chemotherapy-induced oral mucositis: prophylaxis or treatment?. Journal of Lasers in Medical Sciences, v. 2, n. 1, p. 12-17, 2011.

FANI, M. M. et al. The effect of the low-level laser on prevention of chemotherapyinduced oral mucositis in patients with acute leukemia. Journal of Dental Lasers, v. 7, n. 1, p. 22, 2013.

KELNER, Natalie; CASTRO, Jurema Freire Lisboa de. Laser de baixa intensidade no tratamento da mucosite oral induzida pela radioterapia: relato de casos clínicos. Rev Bras Cancerol, v. 53, n. 1, p. 29-33, 2007.

KHOURI, Vivian Youssef et al. Use of therapeutic laser for prevention and treatment of oral mucositis. Brazilian Dental Journal, v. 20, n. 3, p. 215-220, 2009.

LIMA, Aline Gouvêa de et al. Efficacy of low-level laser therapy and aluminum hydroxide in patients with chemotherapy and radiotherapy-induced oral mucositis. Brazilian Dental Journal, v. 21, n. 3, p. 186-192, 2010.

LINO, Maíra Dória Martinez da Costa et al. Laser phototherapy as a treatment for radiotherapy-induced oral mucositis. Brazilian Dental Journal, v. 22, n. 2, p. 162-165, 2011.

LOPES, Carlos de Oliveira; MAS, Josepa Rigau I.; ZÂNGARO, Renato Amaro.

Prevenção da xerostomia e da mucosite oral induzidas por radioterapia com uso do laser de baixa potência. Radiol. Bras, v. 39, n. 2, p. 131-136, 2006.

LOPEZ, Talita Christine Camilo et al. Effect of laser phototherapy in the prevention and treatment of chemo-induced mucositis in hamsters. Braz. Oral Res., São Paulo, v. 27, n. 4, p. 342-348, 2013.

LOWE, A.S.; WALKER, M.D.; O'BYRNE, M; BAXTER, G.D.; HIRST, D.G. Effect of low intensity monochromatic light therapy $(890 \mathrm{~nm})$ on a radiation impaired, wound healing model in murine skin. Lasers Surg Med 1998; v. 23, n. 5, p. 291-298. 
MAIYA, G. Arun et al. Effect of low level helium-neon (He-Ne) laser therapy in the prevention \& treatment of radiation induced mucositis in head \& neck cancer patients. Indian Journal of Medical Research, v. 124, n. 4, p. 399, 2006.

MATOS, J. A; CASTRO, J. F. L. Avaliação de Pacientes Submetidos ao Laser de 830nm Associado ao Protocolo de adequação do Meio Bucal com Gluconato de Clorexidina $0,12 \%$ no Tratamento da Mucosite Oral. Anais do Congresso de Iniciação Científica da Universidade Federal de Pernambuco, 2008. Disponível em: <https://www.ufpe.br/conic/anais>. Acesso em 24 de julho 2014.

MEDEIROS, Niedson Jose de Siqueira et al. Low-power laser therapy in chemicalinduced oral mucositis: a case study. Brazilian Journal of Otorhinolaryngology, v. 79, n. 6, p. 792-792, 2013.

RIBEIRO JÚNIOR, Ophir; BORBA, Alexandre Meireles; GUIMARÃES JÚNIOR, Jayro. Prevenção e tratamento da mucosite bucal: o papel fundamental do cirurgião-dentistaRevisão. Rev. Clín. Pesq. Odontol. (Impr.), v. 6, n. 1, p. 57-62, 201.

SANDOVAL, Renata Lazari et al. Management of chemo-and radiotherapy induced oral mucositis with low-energy laser: initial results of AC Camargo Hospital. Journal of Applied Oral Science, v. 11, n. 4, p. 337-341, 2003.

SANTOS, Paulo Sérgio da Silva et al. Mucosite oral: perspectivas atuais na prevenção e tratamento. RGO - Revista Gaúcha de Odontologia, v. 57, n. 3, 2009.

SANTOS, Paulo Sérgio et al. Prevenção da mucosite oral utilizando LASER terapêutico. Arquivos médicos dos Hospitais e da Faculdade de Ciências Médicas da Santa Casa de São Paulo, v. 55, n. 1, p. 7-11, 2010.

TROTTI, A., BELLM, L.A.; EPSTEIN, J.B.; FRAME, D; FUCHS, H.J.; GWEDE, C.K., et al. Mucositis incidence, severity and associated outcomes in patients with head and neck cancer receiving radiotherapy with or without chemotherapy: a systematic literature review. Radiother Oncol. 2003; v. 66, n. 3, p. 253-262.

WALSH, L. J. The current status of low level laser therapy in dentistry, Part 1. Soft tissue applications. Australian Dental Journal, v. 42, n. 4, p. 247-254, 1997. 